\title{
Are low-energy data already hinting at five dimensions?
}

\author{
Naoyuki Haba, ${ }^{1}$ Nobuchika Okada $\odot,{ }^{2}$ and Toshifumi Yamada $\oplus^{1}$ \\ ${ }^{1}$ Institute of Science and Engineering, Shimane University, Matsue 690-8504, Japan \\ ${ }^{2}$ Department of Physics and Astronomy, University of Alabama, Tuscaloosa, Alabama 35487, USA
}

(Received 18 May 2021; accepted 3 January 2022; published 14 January 2022)

\begin{abstract}
Low-energy data, combined with renormalization group equations, can predict new physics at far higher energy scales. In this paper, we consider the possibility that the measured Higgs boson mass and top quark mass hint at a five-dimensional gauge-Higgs unification (5D GHU) model at a scale above TeV. We note that the vanishing of the Higgs quartic coupling and the proximity of the top quark Yukawa coupling and weak gauge coupling at high scales, inferred from the experimental data, are in harmony with 5D GHU, because in 5D GHU models the Higgs quartic coupling is forbidden by the 5D gauge symmetry and the Yukawa couplings and the weak gauge coupling originate from a common 5D gauge coupling. Based on the above insight, we propose a 5D GHU model where the Standard Model fermions are embedded in 5D fermions in a way to tightly relate the top Yukawa coupling with the weak gauge coupling. Also, the model predicts the presence of vectorlike fermions (other than the Kaluza-Klein modes), which can affect the renormalization group evolutions of the 4D theory and reconcile the scale of vanishing Higgs quartic coupling and that of equality of the top Yukawa and weak gauge couplings, thereby achieving a successful matching of the 4D theory with 5D GHU. We predict the vectorlike fermion mass and the compactification scale of 5D GHU from the conditions for the successful matching.
\end{abstract}

DOI: $10.1103 /$ PhysRevD.105.015018

\section{INTRODUCTION}

In the Standard Model (SM), the Higgs quartic coupling exhibits an interesting property that it vanishes at an energy scale below the Planck scale along renormalization group (RG) evolutions. This property is usually interpreted as implying metastability of our vacuum [1] as the Higgs quartic coupling turns negative above that scale. Another interpretation [2-5] is that the SM is matched, around the scale of vanishing Higgs quartic coupling, with a model of gauge-Higgs unification [6-11] $]^{1}$ in five dimensions (5D GHU), where the SM Higgs field is identified with the fifthdimensional component of a gauge field whose quartic coupling is forbidden by the 5D gauge symmetry.

Although less noted, the top quark Yukawa coupling and the weak gauge coupling also show an interesting feature that they become equal at the energy scale of $O\left(10^{8}\right) \mathrm{GeV}$. This suggests that the two couplings may have a common origin in some new physics above that scale. Indeed, in 5D GHU, the SM Yukawa interactions are part of an extension

\footnotetext{
${ }^{1}$ For early work on extradimensional models, see, e.g., Refs. [12,13].

Published by the American Physical Society under the terms of the Creative Commons Attribution 4.0 International license. Further distribution of this work must maintain attribution to the author(s) and the published article's title, journal citation, and DOI. Funded by SCOAP ${ }^{3}$.
}

of the weak interaction and the Yukawa couplings are tied with the weak gauge coupling.

Motivated by the above insights, we propose a model in which the scale where the Higgs quartic coupling vanishes is reconciled with the scale where the top quark Yukawa coupling equals the weak gauge coupling, and this common scale is interpreted as the scale where 5D GHU emerges.

Our model is characterized by the direct embedding of the SM fermions into 5D bulk fermions. Here, we introduce $5 \mathrm{D}$ fermions in 3 representation of $S U(3)_{W}$ gauge group that extends the $S U(2)_{W}$ weak gauge group. After orbifold compactification, one 5D fermion yields one isospindoublet and one isospin-singlet 4D fermions of opposite chiralities as the massless modes, which are directly identified with an isospin-doublet and an isospin-singlet SM quarks or leptons. Consequently, all the SM Yukawa couplings are identical with the weak gauge coupling at this stage. Small Yukawa couplings other than the top quark Yukawa coupling are reproduced by introducing 4D vectorlike fermions at an orbifold fixed point that mix with the massless modes of 5D fermions. ${ }^{2}$ The above structure materializes the idea that the Yukawa couplings and the

\footnotetext{
${ }^{2}$ In the next section, we will show that it is the square sum of the up-type and down-type quark Yukawa matrices that is related to the weak gauge coupling. Hence, the bottom quark Yukawa coupling can be small without help of the additional 4D vectorlike fermions.
} 
weak gauge coupling are basically the same entity and this fact is encoded in the value of the top quark Yukawa coupling. Thus, this structure is aligned with our motivation to interpret the scale of equality of the top Yukawa coupling and weak gauge coupling, as the scale of 5D GHU.

Another characteristic of our model is the presence of vectorlike fermions (other than Kaluza-Klein modes) that is not arbitrary but is required by the model structure. They are important not only theoretically, but also phenomenologically; since their mass can be smaller than the compactification scale, they can change the RG evolutions of the 4D theory and achieve its matching with the 5D GHU theory. In fact, they can amend a discrepancy in the SM between the scale of equality of the top Yukawa and weak gauge couplings and the scale of vanishing Higgs quartic coupling, thereby making the matching of the 4D and 5D theories successful.

It should be noted that our model is not intended to solve the hierarchy problem of the Higgs mass, and the compactification scale can be much larger than $\mathrm{TeV}$ scale.

Previously, the unification of the weak gauge coupling and top quark Yukawa coupling in a high-scale 5D GHU model has been discussed in Ref. [14] but only in a toy model. We in this paper investigate the unification of the two couplings in a complete, realistic model, and further relate the unification with the high-scale vanishing of the Higgs quartic coupling.

This paper is organized as follows. In Sec. II, we present our model. In Sec. III, we determine the mass of the vectorlike fermions and the compactification scale of $5 \mathrm{D}$ GHU by solving the RG equations of the 4D theory made of the SM content and the vectorlike fermions and matching it with the 5D GHU theory. Section IV summarizes the paper.

\section{MODEL}

We consider $S U(3)_{C} \times S U(3)_{W} \times U(1)_{V}$ gauge theory in a flat $5 \mathrm{D}$ spacetime whose fifth dimension is compactified on $S^{1} / Z_{2}$ orbifold. We write the fifth coordinate as $y$, and the $S^{1}$ is obtained by the identification of $y$ with $y+2 \pi R$. The $S^{1} / Z_{2}$ orbifold is then obtained by identifying $y$ with $-y . S U(3)_{C}$ is the SM QCD gauge group, and $S U(3)_{W} \times U(1)_{V}$ incorporates the electroweak gauge groups. We denote the $S U(3)_{W}$ gauge field by $\left(W_{\mu}, W_{5}\right)$ and the $U(1)_{V}$ gauge field by $\left(V_{\mu}, V_{5}\right)$, where $\mu=0,1,2,3$. The fields transform under the $Z_{2}$ as

$$
\begin{aligned}
W_{\mu}(y) & =P W_{\mu}(-y) P^{\dagger}, \quad W_{5}(y)=-P W_{5}(-y) P^{\dagger}, \\
V_{\mu}(y) & =V_{\mu}(-y), \quad V_{5}(y)=-V_{5}(-y), \\
P & =\left(\begin{array}{ccc}
-1 & 0 & 0 \\
0 & -1 & 0 \\
0 & 0 & 1
\end{array}\right),
\end{aligned}
$$

and accordingly $S U(3)_{W} \times U(1)_{V}$ is broken into $S U(2)_{W} \times U(1)_{W} \times U(1)_{V}$ at $y=0, \pi R$. Gauge group $U(1)_{W} \times U(1)_{V}$ is further broken into SM hypercharge $U(1)_{Y}$ by a brane-localized scalar field in $\left(\mathbf{1}, \mathbf{1},-\frac{1}{2 \sqrt{3}}, \frac{1}{2 \sqrt{3}}\right)$ representation of $S U(3)_{C} \times S U(2)_{W} \times U(1)_{W} \times U(1)_{V}$, denoted by $\phi$, that develops a vacuum expectation value (VEV). Accordingly, the hypercharge $Q_{Y}$ is given in terms of the $U(1)_{W}$ charge $Q_{W}$ and the $U(1)_{V}$ charge $Q_{V}$ by

$$
Q_{Y}=\frac{1}{\sqrt{3}}\left(Q_{W}+Q_{V}\right) .
$$

The four-dimensional components of the $S U(2)_{W} \times U(1)_{Y}$ gauge fields have massless modes, which are identified with the SM electroweak gauge fields. The fifth-dimensional component of the $S U(3)_{W} /\left(S U(2)_{W} \times U(1)_{W}\right)$ gauge field has massless Kaluza-Klein (KK) mode, which is identified with the SM Higgs field, $H$, as

$$
\left.W_{5}\right|_{\text {massless KK }}=\frac{1}{\sqrt{2}}\left(\begin{array}{cc}
O & H \\
H^{\dagger} & 0
\end{array}\right) .
$$

We introduce three copies of four bulk 5D fermions in $\left(\overline{\mathbf{3}}, \mathbf{3},-\frac{1}{\sqrt{3}}\right),(\mathbf{3}, \mathbf{3}, 0),\left(\mathbf{1}, \mathbf{3},-\frac{2}{\sqrt{3}}\right),\left(\mathbf{1}, \mathbf{3}, \frac{1}{\sqrt{3}}\right)$ representations of $S U(3)_{C} \times S U(3)_{W} \times U(1)_{V}$, denoted by $\Psi_{u^{c}}^{i}, \Psi_{d}^{i}, \Psi_{e}^{i}$, $\Psi_{\nu^{c}}^{i}$ with $i=1,2,3$. The three copies correspond to the three generations of the SM. The 5D fermions transform under the $Z_{2}$ as ${ }^{3}$

$$
\begin{gathered}
\Psi_{u^{c}}^{i}(-y)=-\gamma_{5} P \Psi_{u^{c}}^{i}(y), \\
\Psi_{d}^{i}(-y)=\gamma_{5} P \Psi_{d}^{i}(y), \\
\Psi_{e}^{i}(-y)=\gamma_{5} P \Psi_{e}^{i}(y), \\
\Psi_{\nu^{c}}^{i}(-y)=-\gamma_{5} P \Psi_{\nu^{c}}^{i}(y) .
\end{gathered}
$$

The 5D fermions are summarized in Table I.

From the $Z_{2}$ charge assignments, we find that the following chiral fields with $S U(3)_{C} \times S U(2)_{W} \times U(1)_{W} \times$ $U(1)_{V}$ charges possess massless KK modes:

Right-handed $\left(\overline{\mathbf{3}}, \mathbf{2}, \frac{1}{2 \sqrt{3}},-\frac{1}{\sqrt{3}}\right)$ component of $\Psi_{u^{c}}^{i}$,

Left-handed $\left(\overline{\mathbf{3}}, \mathbf{1},-\frac{1}{\sqrt{3}},-\frac{1}{\sqrt{3}}\right)$ component of $\Psi_{u^{c}}^{i}$,

Left-handed $\left(\mathbf{3}, \mathbf{2}, \frac{1}{2 \sqrt{3}}, 0\right) \quad$ component of $\Psi_{d}^{i}$,

\footnotetext{
${ }^{3}$ Since $\Psi_{u^{c}}^{i}, \Psi_{d}^{i}, \Psi_{e}^{i}, \Psi_{\nu^{c}}^{i}$ are in 3 representation of $S U(3)_{W}$, the same $P$ as Eq. (1) enters Eqs. (4)-(7).
} 
TABLE I. The gauge and $Z_{2}$ charges of the 5D fermions. $i$ is the flavor index with $i=1,2,3$.

\begin{tabular}{lcccc}
\hline \hline & $S U(3)_{C}$ & $S U(3)_{W}$ & $U(1)_{V}$ & $Z_{2}$ transformation \\
\hline$\Psi_{u^{c}}^{i}$ & $\overline{\mathbf{3}}$ & $\mathbf{3}$ & $-\frac{1}{\sqrt{3}}$ & $\Psi_{u^{c}}^{i}(-y)=-\gamma_{5} P \Psi_{u^{c}}^{i}(y)$ \\
$\Psi_{d}^{i}$ & $\mathbf{3}$ & $\mathbf{3}$ & 0 & $\Psi_{d}^{i}(-y)=\gamma_{5} P \Psi_{d}^{i}(y)$ \\
$\Psi_{e}^{i}$ & $\mathbf{1}$ & $\mathbf{3}$ & $-\frac{2}{\sqrt{3}}$ & $\Psi_{e}^{i}(-y)=\gamma_{5} P \Psi_{e}^{i}(y)$ \\
$\Psi_{\nu^{i}}^{i}$ & $\mathbf{1}$ & $\mathbf{3}$ & $\frac{1}{\sqrt{3}}$ & $\Psi_{\nu^{c}}^{i}(-y)=-\gamma_{5} P \Psi_{\nu^{c}}^{i}(y)$ \\
\hline \hline
\end{tabular}

Right-handed $\left(\mathbf{3}, \mathbf{1},-\frac{1}{\sqrt{3}}, 0\right)$ component of $\Psi_{d}^{i}$,

Left-handed $\left(\mathbf{1}, 2, \frac{1}{2 \sqrt{3}},-\frac{2}{\sqrt{3}}\right)$ componentof $\Psi_{e}^{i}$,

Right-handed $\left(\mathbf{1}, \mathbf{1},-\frac{1}{\sqrt{3}},-\frac{2}{\sqrt{3}}\right)$ componentof $\Psi_{e}^{i}$,

Right-handed $\left(\mathbf{1}, \mathbf{2}, \frac{1}{2 \sqrt{3}}, \frac{1}{\sqrt{3}}\right)$ component of $\Psi_{\nu^{c}}^{i}$,

Left-handed $\left(\mathbf{1}, \mathbf{1},-\frac{1}{\sqrt{3}}, \frac{1}{\sqrt{3}}\right)$ component of $\Psi_{\nu^{c}}^{i}$.

At this stage, there are chiral anomalies with respect to the $U(1)_{V}$ gauge group. These anomalies should be canceled, and to achieve the cancellation, we introduce 4D chiral fermions of Table II at an orbifold fixed point $y=0$ :

The presence of $4 \mathrm{D}$ fermions $\psi_{q R}^{i}, \psi_{\ell R}^{i}$ is probably a consequence of more fundamental physics that underlies the orbifolding. In the present paper, however, we adhere to the orbifold picture and introduce the $4 \mathrm{D}$ fermions by hand.

The most generic $5 \mathrm{D}$ action is

$$
\begin{aligned}
S= & \int \mathrm{d}^{4} x \int_{-\pi R}^{\pi R} \mathrm{~d} y-\frac{1}{2} \operatorname{tr}\left[W_{M N} W^{M N}\right]-\frac{1}{4} V_{M N} V^{M N} \\
& +i \bar{\Psi}_{u^{c}}^{i} \Gamma^{M} D_{M} \Psi_{u^{c}}^{i}+i \bar{\Psi}_{d}^{i} \Gamma^{M} D_{M} \Psi_{d}^{i}+i \bar{\Psi}_{e}^{i} \Gamma^{M} D_{M} \Psi_{e}^{i} \\
& +i \bar{\Psi}_{\nu^{c}}^{i} \Gamma^{M} D_{M} \Psi_{\nu^{c}}^{i}+\delta(y)\left[i \psi_{q R}^{i \dagger} \bar{\sigma}^{\mu} D_{\mu} \psi_{q R}^{i}+i \psi_{\ell R}^{i \dagger} \bar{\sigma}^{\mu} D_{\mu} \psi_{\ell R}^{i}\right. \\
& -\psi_{q R}^{i T}\left\{\left.A_{i j} \phi i \sigma_{2} \Psi_{u^{c}}^{j}\right|_{R}+\left.B_{i j} \phi^{\dagger} \Psi_{d}^{j}\right|_{L} ^{*}\right\} \\
& \left.-\psi_{\ell R}^{i T}\left\{\left.C_{i j} \phi^{\dagger} \Psi_{e}^{j}\right|_{L} ^{*}+\left.E_{i j} \phi i \sigma_{2} \Psi_{\nu^{c}}^{j}\right|_{R}\right\}+\text { H.c. }\right],
\end{aligned}
$$

where spacetime indices $M, N$ run as $M, N=0,1,2,3,5$, and $D_{M}, D_{\mu}$ denote gauge-covariant derivatives. $\left.\Psi_{{ }^{c}}^{j}\right|_{R}$,

TABLE II. The gauge charges and chirality of the 4D fermions localized at $y=0 . i$ is the flavor index with $i=1,2,3$.

\begin{tabular}{cccccc}
\hline \hline & $S U(3)_{C}$ & $S U(2)_{W}$ & $U(1)_{W}$ & $U(1)_{V}$ & Chirality \\
\hline$\psi_{q R}^{i}$ & $\mathbf{3}$ & $\mathbf{2}$ & 0 & $\frac{1}{2 \sqrt{3}}$ & Right-handed \\
$\psi_{\ell R}^{i}$ & $\mathbf{1}$ & $\mathbf{2}$ & 0 & $-\frac{3}{2 \sqrt{3}}$ & Right-handed \\
\hline \hline
\end{tabular}

$\left.\Psi_{d}^{j}\right|_{L},\left.\Psi_{e}^{j}\right|_{L},\left.\Psi_{\nu^{c}}^{j}\right|_{R}$ denote the 4D-decomposed components of the 5D bulk fermions with the indicated chirality that are in $S U(2)_{W}$ doublet representation, listed in Eqs. (8), (10), (12), (14). $A_{i j}, B_{i j}, C_{i j}, E_{i j}$ denote their coupling constants with 4D localized fermions $\psi_{q R}^{i}, \psi_{\ell R}^{i}$ and the $\left[U(1)_{W} \times U(1)_{V}\right]$-breaking scalar $\phi$. After $\phi$ develops a VEV, the fourth line of Eq. (16) yields a quark mass matrix, which can be recast by a flavor rotation of $\psi_{q R}^{i}$ into

$$
\psi_{q R}^{T}\left(\begin{array}{ll}
M_{3 \times 3} & O_{3 \times 3}
\end{array}\right)\left(\begin{array}{cc}
U_{1} & U_{2} \\
U_{3} & U_{4}
\end{array}\right)\left(\begin{array}{c}
\left.i \sigma_{2} \Psi_{u^{c}}\right|_{R} \\
\left.\Psi_{d}\right|_{L} ^{*}
\end{array}\right),
$$

where $M_{3 \times 3}$ is a $3 \times 3$ diagonal matrix, $O_{3 \times 3}$ denotes the $3 \times 3$ null matrix, $\left(\begin{array}{c}U_{1} U_{2} \\ U_{3} U_{4}\end{array}\right)$ is a unitary matrix, and $U_{1}, U_{2}$, $U_{3}, U_{4}$ are its $3 \times 3$ submatrices. We see that the fields below are massless chiral modes,

$$
\left.\left(U_{3}\right)_{k j} i \sigma_{2} \Psi_{u^{c}}^{j}\right|_{R}+\left.\left(U_{4}\right)_{k j} \Psi_{d}^{j}\right|_{L} ^{*} \equiv Q_{L}^{k} .
$$

$Q_{L}^{k}$ are identified with the SM isospin-doublet quarks. [Remember that $\left.\Psi_{u^{c}}^{j}\right|_{R},\left.\Psi_{d}^{j}\right|_{L} ^{*}$ have hypercharge $Q_{Y}=1 / 6$ after the breaking of $U(1)_{W} \times U(1)_{V}$ into $U(1)_{Y}$.] There also are vectorlike modes with mass $M_{3 \times 3}$ that comprise

$\psi_{q R}^{k} \quad$ and $\left.\quad\left(U_{1}\right)_{k j} i \sigma_{2} \Psi_{u^{c}}^{j}\right|_{R}+\left.\left(U_{2}\right)_{k j} \Psi_{d}^{j}\right|_{L} ^{*} \equiv X_{q L}^{k}$.

They represent new, vectorlike quarks that have the same charges as the SM isospin-doublet quarks. Other fields $\left.\Psi_{u^{c}}^{j}\right|_{L},\left.\Psi_{d}^{j}\right|_{R}$ are identified with the SM up-type and downtype isospin-singlet quarks.

The presence of the vectorlike quarks Eq. (19) is an important prediction of the model. As for theoretical aspects, their presence is not arbitrary but is required by the model structure. Accordingly, their number and gauge charges are uniquely fixed. As for phenomenological aspects, since their mass $M_{3 \times 3}$ can be smaller than the compactification scale, they can modify the RG evolutions of SM parameters. This modification can fix the discrepancy between the scale of vanishing Higgs quartic coupling and that of equality of the top Yukawa and weak gauge couplings, achieving the matching of the 4D theory with the 5D GHU theory.

The quark Yukawa interactions are derived from the 5D $S U(3)_{W}$ gauge interaction as

$$
\begin{aligned}
& S \supset 2 \pi R \int \mathrm{d}^{4} x i \frac{g_{W}}{\sqrt{2}} H\left\{\left.\Psi_{u^{c}}^{i}\right|_{L} ^{\dagger} i \sigma_{2}\left(U_{3}^{\dagger}\right)_{i k} Q_{L}^{\mathrm{k} *}+\left.\Psi_{d}^{i}\right|_{R} ^{T}\left(U_{4}^{\dagger}\right)_{i k} Q_{L}^{\mathrm{k} *}\right\} \\
& \quad+\text { H.c., }
\end{aligned}
$$

where $g_{W}$ denotes the $S U(3)_{W}$ gauge coupling. The up-type and down-type quark Yukawa matrices, $Y_{u}, Y_{d}$, are extracted as $Y_{u}=i \frac{g_{W}}{\sqrt{2}} U_{3}^{*}, Y_{d}=-i \frac{g_{W}}{\sqrt{2}} U_{4}^{*}$. Unfortunately, 
TABLE III. 4D vectorlike fermions localized at $y=0$. Index $a$ runs as $a=1,2$.

\begin{tabular}{ccccc}
\hline \hline & $S U(3)_{C}$ & $S U(2)_{W}$ & $U(1)_{W}$ & $U(1)_{V}$ \\
\hline$\chi_{u}^{a}$ & $\mathbf{3}$ & $\mathbf{1}$ & $\frac{1}{\sqrt{3}}$ & $\frac{1}{\sqrt{3}}$ \\
$\chi_{d}^{a}$ & $\mathbf{3}$ & $\mathbf{1}$ & $-\frac{1}{\sqrt{3}}$ & 0 \\
\hline \hline
\end{tabular}

these Yukawa matrices are unrealistic because the unitarity relation $U_{3}^{\dagger} U_{3}+U_{4}^{\dagger} U_{4}=\operatorname{diag}(1,1,1)$ gives $Y_{u}^{\dagger} Y_{u}+Y_{d}^{\dagger} Y_{d}=$ $\frac{g_{W}^{2}}{2} \operatorname{diag}(1,1,1)$, which contradicts the smallness of the first and second generation Yukawa couplings. To reproduce their smallness, we introduce two generations of 4D vectorlike fermions of Table III at $y=0$.

$4 \mathrm{D}$ vectorlike fermions $\chi_{u}^{a}, \chi_{d}^{a}$ mix with the massless $\mathrm{KK}$ modes of $5 \mathrm{D}$ fermions through the term

$$
\begin{aligned}
-\Delta S= & \int \mathrm{d}^{4} x \int_{-\pi R}^{\pi R} \mathrm{~d} y \delta(y)\left[\left.\left.M_{u}^{a j} \chi_{u}^{a}\right|_{L} ^{T} i \sigma_{2} \Psi_{u^{c}}^{j}\right|_{L}\right. \\
& \left.+\left.\left.M_{d}^{a j} \chi_{d}^{a}\right|_{L} ^{\dagger} \Psi_{d}^{j}\right|_{R}+\text { H.c. }+\mu_{u}^{a} \bar{\chi}_{u}^{a} \chi_{u}^{a}+\mu_{d}^{a} \bar{\chi}_{d}^{a} \chi_{d}^{a}\right],
\end{aligned}
$$

which reduces the Yukawa couplings of two generations by the ratio $\mu_{u} / M_{u}$ or $\mu_{d} / M_{d}$.

Even with the above mechanism to reduce the first and second generation Yukawa couplings, there is a tight connection between the third generation Yukawa couplings and the $S U(3)_{W}$ gauge coupling. Since the relation $Y_{u}^{\dagger} Y_{u}+Y_{d}^{\dagger} Y_{d}=\frac{g_{W}^{2}}{2} \operatorname{diag}(1,1,1)$ is still valid for the third generation quarks, by neglecting the mixing between the third generation and other generation quarks, we get

$$
y_{t}^{2}+y_{b}^{2} \simeq \frac{1}{2} g_{W}^{2},
$$

where $y_{t}, y_{b}$ denote the top and bottom quark Yukawa couplings. As $y_{t}$ is dominantly large, and $g_{W}$ matches with the weak gauge coupling, Eq. (22) materializes the idea that equality of the top quark Yukawa coupling and the weak gauge coupling hints at 5D GHU.

As for the lepton sector, the charged lepton Yukawa matrix and the neutrino Dirac Yukawa matrix are derived in the same fashion. The fourth line of Eq. (16) gives a lepton mass matrix, which can be recast into the form

$$
\psi_{\ell R}^{T}\left(\begin{array}{ll}
M_{3 \times 3}^{\prime} & O_{3 \times 3}
\end{array}\right)\left(\begin{array}{cc}
V_{1} & V_{2} \\
V_{3} & V_{4}
\end{array}\right)\left(\begin{array}{c}
\left.\Psi_{e}\right|_{L} ^{*} \\
\left.i \sigma_{2} \Psi_{\nu^{c}}\right|_{R}
\end{array}\right),
$$

where $M_{3 \times 3}^{\prime}$ is a $3 \times 3$ diagonal matrix, $\left(\begin{array}{ll}V_{1} & V_{2} \\ V_{3} & V_{4}\end{array}\right)$ is a unitary matrix, and $V_{1}, V_{2}, V_{3}, V_{4}$ are its $3 \times 3$ submatrices. The massless chiral modes below

$$
\left.\left(V_{3}\right)_{k j} \Psi_{e}^{j}\right|_{L} ^{*}+\left.\left(V_{4}\right)_{k j} j \sigma_{2} \Psi_{\nu^{c}}^{j}\right|_{R} \equiv L_{L}^{k}
$$

are identified with the SM isospin-doublet leptons. The vectorlike modes with mass $M_{3 \times 3}^{\prime}$ that comprise

$$
\begin{aligned}
& \psi_{\ell R}^{k}, \\
& \left(V_{1}\right)_{k j}\left(\left.\Psi_{e}^{j}\right|_{L}\right)^{*}+\left.\left(V_{2}\right)_{k j} i \sigma_{2} \Psi_{\nu^{c}}^{j}\right|_{R} \equiv X_{\ell L}^{\mathrm{k} *}
\end{aligned}
$$

represent new, vectorlike leptons, which have the same charges as the SM isospin-doublet leptons. Other fields $\left.\Psi_{e}^{j}\right|_{R},\left.\Psi_{\nu^{c}}^{j}\right|_{L}$ are identified with the SM charged leptons and isospin-singlet neutrinos, respectively. The realistic charged lepton Yukawa couplings and tiny active neutrino masses are obtained by introducing vectorlike fermions analogous to those of Table III.

As with the vectorlike quarks, the presence of the vectorlike leptons Eq. (25) is an important prediction of the model.

\section{ESTIMATION OF VECTORLIKE FERMION MASS AND COMPACTIFICATION SCALE}

The presence of vectorlike quarks Eq. (19) and vectorlike leptons Eq. (25) can modify the RG evolutions of SM parameters and amend the discrepancy between the scale of vanishing Higgs quartic coupling and that of equality of the top Yukawa and weak gauge couplings, enabling us to match the 4D theory with the 5D GHU theory. Noting this fact, we estimate the mass of the vectorlike fermions with which the matching conditions of the 4D theory with the 5D GHU theory are satisfied. Additionally, we obtain the compactification scale from the matching scale.

The matching relates the radiatively generated potential for the fifth-dimensional component of $S U(3)_{W}$ gauge boson in 5D GHU with the Higgs potential in the 4D theory. It also relates the $S U(3)_{W}$ gauge coupling in the former with the weak gauge coupling and quark Yukawa couplings in the latter. For the Higgs potential, we adopt the general result [2] that the scale of vanishing Higgs quartic coupling $\lambda$ at one loop equals the compactification scale of 5D GHU models as

$$
\lambda\left(\frac{1}{2 \pi R}\right)=0 .
$$

Note that Eq. (26) has been derived by considering oneloop threshold corrections from all the Kaluza-Klein modes of the $S U(3)_{W}$ gauge field and bulk fermions in a general 5D GHU model. Hence, we can use Eq. (26) without further including threshold corrections from Kaluza-Klein modes. Note also that the 4D fermions localized at $y=0$ do not alter Eq. (26) at one-loop level because these fermions do not couple to the Higgs field at tree level. For the weak gauge coupling and quark Yukawa couplings, we perform a tree-level matching around the compactification scale as 


$$
\begin{aligned}
g_{W} & =g_{S U(2)_{W}}, \\
\frac{1}{2} g_{W}^{2} & =y_{t}^{2}+y_{b}^{2}, \quad \text { at } \mu=\frac{1}{2 \pi R},
\end{aligned}
$$

where $g_{S U(2)_{W}}$ denotes the weak gauge coupling, and the second condition is based on Eq. (22).

We numerically estimate the value of the vectorlike fermion mass with which Eqs. (26), (27) are simultaneously satisfied at one unique scale and the matching is successful. This scale is then identified with the compactification scale $1 /(2 \pi R)$. To this end, we solve the two-loop RG equations [15-17] of the 4D theory (comprising the SM content and the vectorlike fermions) by varying the vectorlike fermion mass. The masses of the vectorlike quarks and leptons are assumed degenerate, for simplicity. As for the SM parameters used as inputs of the RG equations, since the top quark pole mass significantly affects the RG evolution of the Higgs quartic coupling, we vary it in the $3 \sigma$ range of the latest measurement of the CMS Collaboration, $170.5 \pm$ $0.8 \mathrm{GeV}[18] .{ }^{4}$ The strong gauge coupling is fixed at the central value of the same measurement, and the other parameters are fixed at their central values in accordance with Ref. [20]. The pole masses of the top quark, Higgs particle, and $W, Z$ particles are translated into the input values of the top Yukawa coupling, the Higgs quartic coupling and the weak mixing angle for the $\mathrm{RG}$ equations by using the code [21], based on the results of [22-28]. In Fig. 1, we give a contour plot of

$$
\log _{10}\left(\mu_{\lambda} / \mu_{g y}\right)
$$

where

$$
\begin{aligned}
\lambda\left(\mu_{\lambda}\right) & =0, \\
\left.\frac{1}{2} g_{S U(2)_{W}}^{2}\right|_{\mu=\mu_{g y}} & =y_{t}^{2}+\left.y_{b}^{2}\right|_{\mu=\mu_{g y}},
\end{aligned}
$$

on the plane of the vectorlike fermion mass $M_{\text {vec }}$ versus the top quark pole mass $M_{t}$.

The contour of $\log _{10}\left(\mu_{\lambda} / \mu_{g y}\right)=0$ is the region where Eqs. (26) and (27) are simultaneously satisfied at one scale and the matching is successful. Thus, this contour is the model's prediction on the vectorlike fermion mass $M_{\mathrm{vec}}$ and the precise top quark pole mass. Since the contour exists only for $M_{t} \gtrsim 171.4 \mathrm{GeV}$, if future measurements of the top quark pole mass exclude this range of $M_{t}$, the present model is falsified. To confirm the model, one should test the relation between the vectorlike fermion mass and the top quark pole mass given by the contour. This is not possible in near-future collider experiments, since the vectorlike

\footnotetext{
${ }^{4}$ The latest measurement of the top quark pole mass of the ATLAS Collaboration is $171.1_{-1.0}^{+1.2} \mathrm{GeV}$ [19], which favors large values compared to the CMS result.
}

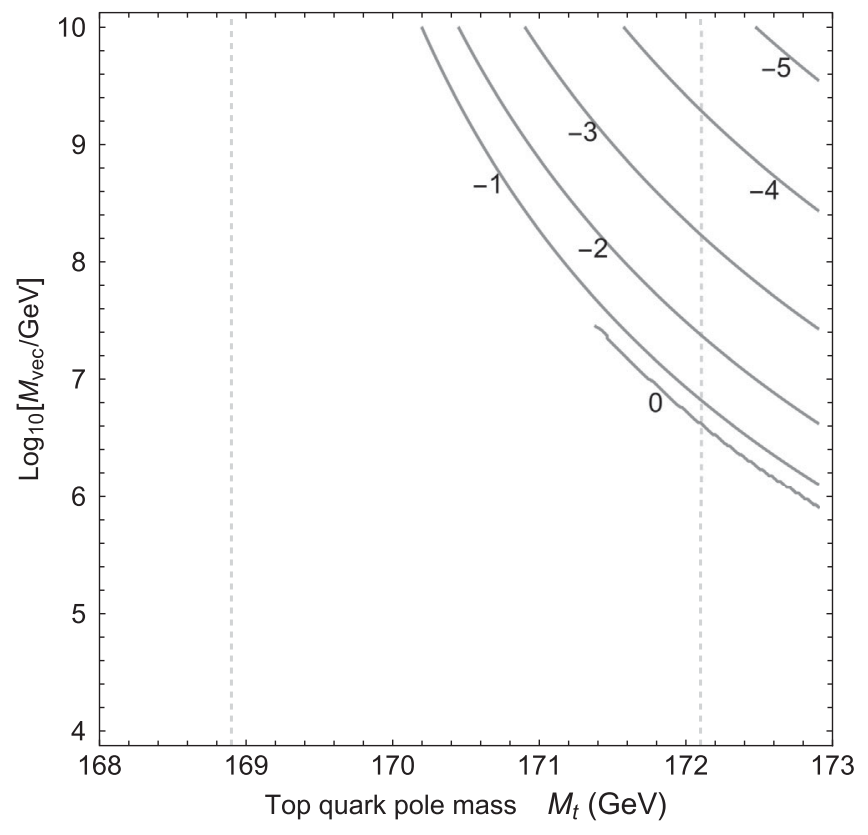

FIG. 1. Contour plot of $\log _{10}\left(\mu_{\lambda} / \mu_{g y}\right)$ Eq. (28), which is the logarithm of the ratio of the scale where the Higgs quartic coupling vanishes and the scale where the quark Yukawa couplings and weak gauge coupling satisfy Eq. (27). The vertical axis is the logarithm of the vectorlike fermion mass $\log _{10}\left(M_{\mathrm{vec}} / \mathrm{GeV}\right)$, and the horizontal axis is the top quark pole mass $M_{t}$. The range of the horizontal axis corresponds to the $3 \sigma$ range of the top quark pole mass measured by the CMS Collaboration, and the range between the two vertical dashed lines corresponds to the $2 \sigma$ range. On the contour of $\log _{10}\left(\mu_{\lambda} / \mu_{\text {gy }}\right)=0$, Eqs. (26) and (27) are simultaneously satisfied at one scale and the matching is successful.

fermion mass is predicted to be above $10^{3} \mathrm{TeV}$. On the contour of $\log _{10}\left(\mu_{\lambda} / \mu_{g y}\right)=0$, the value of $\mu_{\lambda}=\mu_{g y}$, which corresponds to the compactification scale $1 /(2 \pi R)$, varies from $10^{11.6} \mathrm{GeV}$ to $10^{10.2} \mathrm{GeV}$ from the upper left to the lower right. The above range is the model's prediction on the compactification scale.

The contour of $\log _{10}\left(\mu_{\lambda} / \mu_{g y}\right)=0$ is interrupted at $M_{t} \simeq$ $171.4 \mathrm{GeV}$ because for lower values of the top quark pole mass, the Higgs quartic coupling remains positive along RG evolutions and hence $\mu_{\lambda}$ is not defined. There are no contours in the area under the contour of $\log _{10}\left(\mu_{\lambda} / \mu_{g y}\right)=0$ because the Higgs quartic coupling remains positive there. The reason that the Higgs quartic coupling tends to remain positive for smaller values of the vectorlike fermion mass is that the presence of the vectorlike fermions increases the weak gauge coupling along RG evolutions, which changes the beta function of the Higgs quartic coupling positive. To visualize the situation, we show in Fig. 2 the plot of $\log _{10}\left(\mu_{\lambda} / \mu_{g y}\right)$ for a fixed value of the top quark pole mass $M_{t}=172.1 \mathrm{GeV}$. One sees that as the vectorlike fermion mass decreases, $\log _{10}\left(\mu_{\lambda} / \mu_{g y}\right)$ increases and becomes about 0 , and then the plot line vanishes because $\mu_{\lambda}$ is not defined for lower values of the vectorlike fermion mass. 


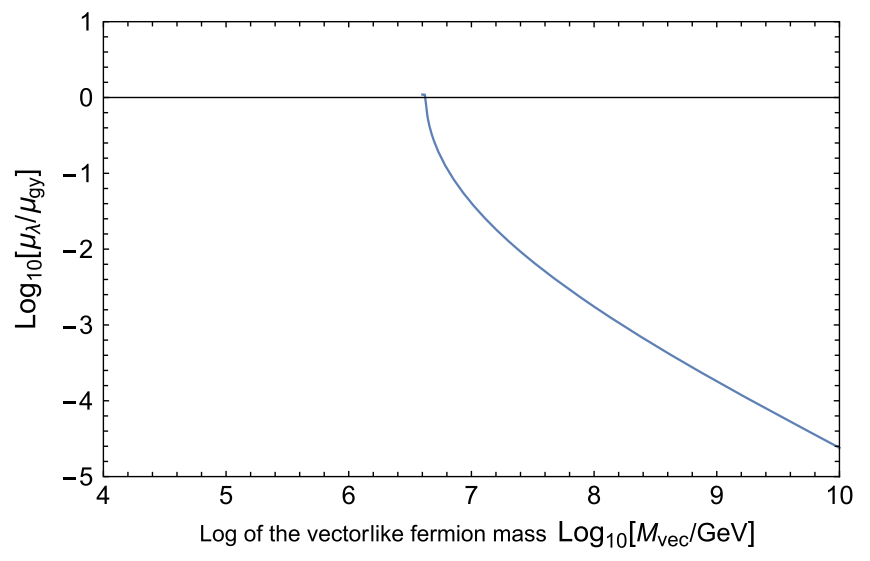

FIG. 2. $\log _{10}\left(\mu_{\lambda} / \mu_{g y}\right)$ versus the logarithm of the vectorlike fermion mass, for a fixed value of the top quark pole mass $M_{t}=172.1 \mathrm{GeV}$. The plot line vanishes for lower values of the vectorlike fermion mass because $\mu_{\lambda}$ is not defined.

Interestingly, those parameter sets that yield $\mu_{\lambda} / \mu_{g y}=1$ are critical in the sense that the Higgs quartic coupling nearly remains positive for such sets. To illustrate this, we present in Fig. 3 the RG evolutions of the Higgs quartic coupling, the square sum of Yukawa couplings, and the weak gauge coupling for a parameter set $\left(M_{t}, \log _{10}\left(M_{\mathrm{vec}} / \mathrm{GeV}\right)\right)=(172.1 \mathrm{GeV}, 6.6)$. The solid purple, blue, and green lines are the RG evolutions of the Higgs quartic coupling, the Yukawa coupling square sum, and half the weak gauge coupling squared, respectively. The dashed lines are their RG evolutions if the 4D theory were valid at high scales. One confirms $\mu_{\lambda} / \mu_{g y}=1$ and that the Higgs quartic coupling nearly remains positive. For such parameter sets, even a slight decrease in the

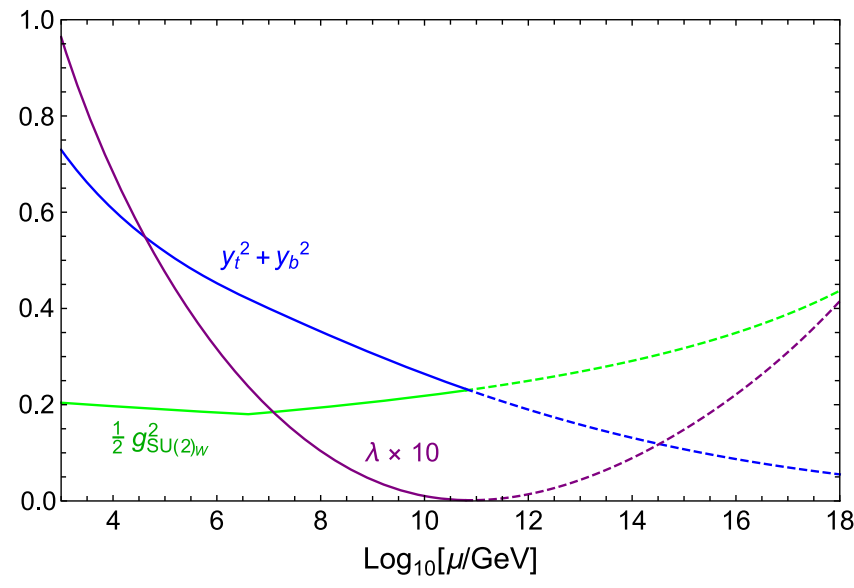

FIG. 3. RG evolutions of the Higgs quartic coupling $\lambda$, the square sum of Yukawa couplings $y_{t}^{2}+y_{b}^{2}$, and half the weak gauge coupling squared $(1 / 2) g_{S U(2)_{W}}^{2}$ for $M_{t}=172.1 \mathrm{GeV}$ and $\log _{10}\left(M_{\mathrm{vec}} / \mathrm{GeV}\right)=6.6$, colored in purple, blue, and green, respectively. $\mu$ denotes the renormalization scale. The solid lines are the RG evolutions in the current model, and the dashed lines are those if the $4 \mathrm{D}$ theory were valid at high scales. vectorlike fermion mass enhances the weak gauge coupling and the Higgs quartic coupling along RG evolutions, rendering the Higgs quartic coupling nonvanishing.

\section{SUMMARY}

The vanishing of the Higgs quartic coupling and the proximity of the top Yukawa and weak gauge couplings at high energy scales hint at 5D GHU. Based on the above idea, we have proposed a model where the scale of vanishing Higgs quartic coupling and equality of the square sum of the top and bottom Yukawa couplings $y_{t}^{2}+y_{b}^{2}$ and half the weak gauge coupling squared $(1 / 2) g_{S U(2)_{W}}^{2}$ is interpreted as the compactification scale of 5D GHU. The model is characterized by the embedding of the SM fermions in 3 representation of the $S U(3)_{W}$ gauge group. This embedding leads to the equality of $y_{t}^{2}+y_{b}^{2}$ and $(1 / 2) g_{S U(2)_{W}}^{2}$ that must be satisfied at the compactification scale. The model structure necessitates the presence of isospin-doublet vectorlike quarks and leptons. These vectorlike fermions modify the RG evolutions of the $4 \mathrm{D}$ theory and reconcile the scale of vanishing Higgs quartic coupling and that where $y_{t}^{2}+y_{b}^{2}=(1 / 2) g_{S U(2)_{W}}^{2}$ holds, thereby achieving the successful matching of the 4D theory with 5D GHU. Based on this property, we have calculated the RG equations of the 4D theory and obtained a prediction for the vectorlike fermion mass, the precise top quark pole mass, and the compactification scale. The prediction for the former two is given by the 0 contour in Fig. 1, and that for the compactification scale varies from $10^{11.6}$ to $10^{10.2} \mathrm{GeV}$ as one moves from the upper left to the lower right on the contour. About experimental testability of the model, if future measurements of the top quark pole mass exclude the region above about $171.4 \mathrm{GeV}$, the model is ruled out. The model can be confirmed by testing the relation between the vectorlike fermion mass and the top quark pole mass given by the 0 contour in Fig. 1, but this is not possible in near-future collider experiments, since the vectorlike fermion mass is predicted to be above $10^{3} \mathrm{TeV}$.

\section{ACKNOWLEDGMENTS}

This work is partially supported by Scientific Grants by the Ministry of Education, Culture, Sports, Science and Technology of Japan: Grants No. 17K05415 and No. 21H000761 (N. H.) and No. 19K147101 (T. Y.), and by the United States Department of Energy Grant No. DESC0012447 (N. O.).

\section{APPENDIX: MATCHING CONDITION FOR THE HIGGS QUARTIC COUPLING}

We rederive the one-loop matching condition for the Higgs quartic coupling in Ref. [2] utilized in the main text, 


$$
\lambda\left(\frac{1}{2 \pi R}\right)=0
$$

First, we study a general $S U(3)_{W}$ gauge theory in a fivedimensional spacetime compactified on $S^{1} / Z_{2}$ orbifold, with 5D bulk fermions in the fundamental representation, and without 4D chiral fermions localized at $y=0$ or $\pi R$. The action with the gauge-fixing term is given by

$$
\begin{aligned}
S= & \int \mathrm{d}^{4} x \int_{-\pi R}^{\pi R} \mathrm{~d} y-\frac{1}{2} \operatorname{tr}\left[W_{M N} W^{M N}\right] \\
& -\frac{1}{\xi} \operatorname{tr}\left[\left(\partial_{\mu} W^{\mu}-\xi \bar{D}_{5} W_{5}\right)^{2}\right] \\
& +2 \operatorname{tr}\left[b\left(\partial_{\mu} D^{\mu}-\xi \bar{D}_{5} D_{5}\right) c\right] \\
& +\frac{i}{2} \bar{\Psi} \Gamma^{M} D_{M} \Psi-\frac{i}{2}\left(D_{M} \bar{\Psi}\right) \Gamma^{M} \Psi
\end{aligned}
$$

where $\xi$ is the gauge-fixing parameter, $b, c$ are ghost fields, and $\Psi$ represents multiple bulk fermions in the 3 representation of $S U(3)_{W}$ gauge group. Also, in the first line, $\bar{D}_{5}$ denotes a covariant derivative whose gauge field part is replaced by the VEV of $W_{5}$ field. We restrict ourselves to the case when $\Psi$ is $Z_{2}$ even, as $Z_{2}$-odd bulk fermions give the same contribution as $Z_{2}$-even ones to the one-loop effective potential. The gauge field, ghost fields, and bulk fermions transform under the $Z_{2}$ as

$$
\begin{aligned}
W_{\mu}(y) & =P W_{\mu}(-y) P^{\dagger}, \quad W_{5}(y)=-P W_{5}(-y) P^{\dagger}, \\
b(y) & =P b(-y) P^{\dagger}, \quad c(y)=P c(-y) P^{\dagger}, \\
\Psi(y) & =\gamma_{5} P \Psi(-y), \\
P & =\left(\begin{array}{ccc}
-1 & 0 & 0 \\
0 & -1 & 0 \\
0 & 0 & 1
\end{array}\right) .
\end{aligned}
$$

The $Z_{2}$ transformation property above breaks $S U(3)_{W}$ into $S U(2)_{W} \times U(1)_{W}$. The massless mode of the $S U(3)_{W} /\left(S U(2)_{W} \times U(1)_{W}\right)$ component of $W_{5}$ is identified with a Higgs field. Unlike Ref. [2], we do not impose any nontrivial condition for the periodic change $y \rightarrow$ $y+2 \pi R$.

We compute the one-loop effective potential for the $W_{5}$ VEV. To this end, we extract the quadratic part of the action Eq. (A1) in the presence of the $W_{5}$ VEV parametrized as

$$
\left\langle W_{5}\right\rangle=\frac{1}{2 g R}\left(\begin{array}{ccc}
0 & 0 & 0 \\
0 & 0 & a \\
0 & a & 0
\end{array}\right) .
$$

The quadratic part is found to be

$$
\begin{aligned}
\left.S\right|_{\text {quad }}= & \int \mathrm{d}^{4} x \int_{-\pi R}^{\pi R} \mathrm{~d} y \operatorname{tr}\left[W_{\mu}\left(\eta^{\mu \nu} \square-\left(1-\frac{1}{\xi}\right) \partial^{\mu} \partial^{\nu}\right) W_{\nu}-W_{\mu} \bar{D}_{5}^{2} W^{\mu}-W_{5} \square W_{5}+\xi W_{5} \bar{D}_{5}^{2} W_{5}+b \square c-\xi b \bar{D}_{5}^{2} c\right] \\
& +i \bar{\Psi}\left(\gamma^{\mu} \partial_{\mu}+i \gamma_{5} \bar{D}_{5}\right) \Psi \\
& +2\left[\int \mathrm{d}^{4} x \operatorname{tr}\left[2\left(\partial^{\mu} W_{\mu}\right) W_{5}+W_{\mu} \bar{D}_{5} W^{\mu}-\xi W_{5} \bar{D}_{5} W_{5}\right]-\bar{\Psi} \gamma_{5} \Psi\right]_{y=0}^{y=\pi R},
\end{aligned}
$$

where $\bar{D}_{5}$ is a covariant derivative whose gauge field part is given by $\left\langle W_{5}\right\rangle$ of Eq. (A3). From the $Z_{2}$ transformation property and the periodicity, the fields obey the following boundary conditions at $y=0, \pi R$ :

$$
\begin{aligned}
W_{\mu}(y) & =P W_{\mu}(y) P^{\dagger}, \quad W_{5}(y)=-P W_{5}(y) P^{\dagger}, \\
\bar{D}_{5} W_{\mu}(y) & =-P \bar{D}_{5} W_{\mu}(y) P^{\dagger}, \quad W_{5}(y)=P \bar{D}_{5} W_{5}(y) P^{\dagger}, \\
b(y) & =P b(y) P^{\dagger}, \quad c(y)=P c(y) P^{\dagger}, \\
\bar{D}_{5} b(y) & =-P \bar{D}_{5} b(y) P^{\dagger}, \quad \bar{D}_{5} c(y)=-P \bar{D}_{5} c(y) P^{\dagger}, \\
\Psi(y) & =\gamma_{5} P \Psi(y), \\
\bar{D}_{5} \Psi(y) & =-\gamma_{5} \bar{D}_{5} P \Psi(y), \quad \text { at } y=0, \pi R .
\end{aligned}
$$

Note that given the above boundary conditions, the boundary action Eq. (A5) vanishes, and the ghost term is Hermite. We calculate the eigenvalues of the quadratic operators in the bulk Eq. (A4) under the condition that the eigenfunctions fulfill the boundary conditions Eq. (A6). The eigenvalue equations are given by

$$
\begin{aligned}
\left(\eta^{\mu \nu} \square-\left(1-\frac{1}{\xi}\right) \partial^{\mu} \partial^{\nu}\right) W_{\nu}-\bar{D}_{5}^{2} W^{\mu} & =\lambda_{W_{\mu}} W^{\mu}, \\
-\square W_{5}+\xi \bar{D}_{5}^{2} W_{5} & =\lambda_{W_{5}} W_{5}, \\
\square c-\xi \bar{D}_{5}^{2} c & =\lambda_{c} c, \\
\square b-\xi \bar{D}_{5}^{2} b & =\lambda_{b} b, \\
\left(i \gamma^{\mu} \partial_{\mu}-\gamma_{5} \bar{D}_{5}\right)^{2} \Psi & =\lambda_{\Psi} \Psi .
\end{aligned}
$$

The solutions to the above equations, before the boundary conditions are imposed, are in the form, 


$$
\begin{aligned}
W_{\mu}= & e^{ \pm i p x} \Omega(y)\left\{C_{\mu}^{T L} \cos (m y)+S_{\mu}^{T L} \sin (m y)\right\} \Omega^{\dagger}(y) \\
& +e^{ \pm i p x} \Omega(y)\left\{C_{\mu}^{S} \cos (m y)+S_{\mu}^{S} \sin (m y)\right\} \Omega^{\dagger}(y), \\
W_{5}= & e^{ \pm i p x} \Omega(y)\left\{C_{5} \cos (m y)+S_{5} \sin (m y)\right\} \Omega^{\dagger}(y), \\
c= & e^{ \pm i p x} \Omega(y)\left\{C_{c} \cos (m y)+S_{c} \sin (m y)\right\} \Omega^{\dagger}(y), \\
b= & e^{ \pm i p x} \Omega(y)\left\{C_{b} \cos (m y)+S_{b} \sin (m y)\right\} \Omega^{\dagger}(y), \\
\Psi= & e^{ \pm i p x} \Omega(y)\left\{C^{L} \cos (m y)+S^{L} \sin (m y)\right\} \\
& +e^{ \pm i p x} \Omega(y)\left\{C^{R} \cos (m y)+S^{R} \sin (m y)\right\},
\end{aligned}
$$

where

$$
\begin{aligned}
\Omega(y) & =\exp \left[i g \int_{0}^{y}\left\langle W_{5}\right\rangle \mathrm{d} y^{\prime}\right] \\
& =\exp \left[\frac{i}{2}\left(\begin{array}{lll}
0 & 0 & 0 \\
0 & 0 & a \\
0 & a & 0
\end{array}\right) \frac{y}{R}\right] .
\end{aligned}
$$

Here $p$ denotes a four-momentum, and $m$ denotes the mass of Kaluza-Klein modes determined from the boundary conditions. The zero-modes correspond to the case where $m=0$. The $C$ s and $S$ s are constants satisfying $p^{\mu} C_{\mu}^{T L}=$ $p^{\mu} S_{\mu}^{T L}=0, C_{\mu}^{S} \propto S_{\mu}^{T L} \propto p_{\mu}, \gamma_{5} C^{L}=-C^{L}, \gamma_{5} S^{L}=-S^{L}$, $\gamma_{5} C^{R}=C^{R}, \gamma_{5} S^{R}=S^{R}$. The corresponding eigenvalues are given by

$$
\begin{aligned}
\lambda_{W_{\mu}^{T L}} & =-p^{2}+m^{2}, \quad \lambda_{W_{\mu}^{S}}=-p^{2} / \xi+m^{2}, \\
\lambda_{W_{5}} & =p^{2}-\xi m^{2}, \\
\lambda_{c} & =-p^{2}+\xi m^{2}, \\
\lambda_{b} & =-p^{2}+\xi m^{2}, \\
\lambda_{\Psi} & =-p^{2}+m^{2} .
\end{aligned}
$$

Now we impose the boundary conditions Eq. (A6) on the solutions Eq. (A8) and determine the value of $m$. The boundary conditions are equivalent to the following constraints on the constants:

$$
\begin{aligned}
P C_{\mu}^{T L} P^{\dagger} & =C_{\mu}^{T L}, \quad P S_{\mu}^{T L} P^{\dagger}=-S_{\mu}^{T L}, \\
P \Omega(\pi R)\left\{C_{\mu}^{T L} \cos (m \pi R)+S_{\mu}^{T L} \sin (m \pi R)\right\} \Omega^{\dagger}(\pi R) P^{\dagger} & =\Omega(\pi R)\left\{C_{\mu}^{T L} \cos (m \pi R)+S_{\mu}^{T L} \sin (m \pi R)\right\} \Omega^{\dagger}(\pi R), \\
P \Omega(\pi R)\left\{-C_{\mu}^{T L} \sin (m \pi R)+S_{\mu}^{T L} \cos (m \pi R)\right\} \Omega^{\dagger}(\pi R) P^{\dagger} & =-\Omega(\pi R)\left\{-C_{\mu}^{T L} \sin (m \pi R)+S_{\mu}^{T L} \cos (m \pi R)\right\} \Omega^{\dagger}(\pi R),
\end{aligned}
$$

(the same for $C_{\mu}^{S}, S_{\mu}^{S}, C_{c}, S_{c}, C_{b}, S_{b}$, and the sign is flipped for $C_{5}, S_{5}$ ),

and

$$
\begin{aligned}
P C^{L} & =-C^{L}, \quad P S^{L}=S^{L}, \\
P \Omega(\pi R)\left\{C^{L} \cos (m \pi R)+S^{L} \sin (m \pi R)\right\} & =-\Omega(\pi R)\left\{C^{L} \cos (m \pi R)+S^{L} \sin (m \pi R)\right\}, \\
P \Omega(\pi R)\left\{-C^{L} \sin (m \pi R)+S^{L} \cos (m \pi R)\right\} & =\Omega(\pi R)\left\{-C^{L} \sin (m \pi R)+S^{L} \cos (m \pi R)\right\}, \\
P C^{R} & =C^{R}, \quad P S^{R}=-S^{R}, \\
P \Omega(\pi R)\left\{C^{R} \cos (m \pi R)+S^{R} \sin (m \pi R)\right\} & =\Omega(\pi R)\left\{C^{R} \cos (m \pi R)+S^{R} \sin (m \pi R)\right\}, \\
P \Omega(\pi R)\left\{-C^{R} \sin (m \pi R)+S^{R} \cos (m \pi R)\right\} & =-\Omega(\pi R)\left\{-C^{R} \sin (m \pi R)+S^{R} \cos (m \pi R)\right\} .
\end{aligned}
$$

In Eq. (A11), nonzero solutions for the set $\left(C_{\mu}^{T L} \cos (m y), S_{\mu}^{T L} \sin (m y)\right)$ exist when

$m=\frac{n}{R}, \quad \frac{n \pm a / 2}{R}, \quad \frac{n \pm a}{R}, \quad(n=0, \pm 1, \pm 2, \ldots)$,

and when $n>0$ there are one solution for $m=n / R$, two solutions for each of $m=\left(n \pm \frac{a}{2}\right) / R$, and one solution for each of $m=(n \pm a) / R$. The solutions when $n<0$ are degenerate with those when $n>0$. When $n=0$, there are one solution for $m=0$, two common solutions for $m= \pm \frac{a}{2} / R$, and one common solution for $m= \pm a / R$, which, respectively, correspond to the photon, $W^{ \pm}$bosons, and $Z$ boson with the wrong Weinberg angle. The values of $m$ and the number of solutions for each $m$ are the same for $\left(C_{\mu}^{S}, S_{\mu}^{S}\right),\left(C_{c}, S_{c}\right),\left(C_{b}, S_{b}\right)$, and $\left(C_{5}, S_{5}\right)$.

In Eq. (A12), nonzero solutions for the set $\left(C^{L} \cos (m y), S^{L} \sin (m y)\right)$ exist when

$$
m=\frac{n}{R}, \quad \frac{n \pm a / 2}{R}, \quad(n=0, \pm 1, \pm 2, \ldots)
$$

and when $n>0$ there are one solution for $m=n / R$ and one solution for each of $m=\left(n \pm \frac{a}{2}\right) / R$. The solutions 
when $n<0$ are degenerate with those when $n>0$. When $n=0$, there are one solution for $m=0$ and one common solution for $m= \pm \frac{a}{2} / R$, which, respectively, correspond to one Weyl fermion that does not have a Yukawa coupling with the Higgs field ${ }^{5}$ and one of a pair of Weyl fermions that have a Yukawa coupling.

Nonzero solutions for the set $\left(C^{R} \cos (m y), S_{R} \sin (m y)\right)$ exist when

$$
m=\frac{n}{R}, \quad \frac{n \pm a / 2}{R} \quad(n=0, \pm 1, \pm 2, \ldots)
$$

and when $n>0$ there are one solution for $m=n / R$ and one solution for each of $m=\left(n \pm \frac{a}{2}\right) / R$. The solutions when $n<0$ are degenerate with those when $n>0$. When $n=0$, there only is one common solution for $m= \pm \frac{a}{2} / R$, which corresponds to one of a pair of Weyl fermions that have a Yukawa coupling.

Substituting Eqs. (A13)-(A15) into Eq. (A10), we obtain the true eigenvalues.

From the eigenvalues and their duplications found above, the one-loop effective potential for the $W_{5} \mathrm{VEV}$ $a$ is computed as

$$
\begin{aligned}
V_{\mathrm{eff}}(a)-V_{\mathrm{eff}}(0)= & -\frac{i}{2} \int \frac{\mathrm{d}^{4} p}{(2 \pi)^{4}} \sum_{n=1}^{\infty}\left[3 \left\{2 \log \frac{-p^{2}+\left(n+\frac{a}{2}\right)^{2} / R^{2}}{-p^{2}+n^{2} / R^{2}}+2 \log \frac{-p^{2}+\left(n-\frac{a}{2}\right)^{2} / R^{2}}{-p^{2}+n^{2} / R^{2}}\right.\right. \\
& \left.+\log \frac{-p^{2}+(n+a)^{2} / R^{2}}{-p^{2}+n^{2} / R^{2}}+\log \frac{-p^{2}+(n-a)^{2} / R^{2}}{-p^{2}+n^{2} / R^{2}}\right\} \\
& \left.-2 N_{\Psi}\left\{2 \log \frac{-p^{2}+\left(n+\frac{a}{2}\right)^{2} / R^{2}}{-p^{2}+n^{2} / R^{2}}+2 \log \frac{-p^{2}+\left(n-\frac{a}{2}\right)^{2} / R^{2}}{-p^{2}+n^{2} / R^{2}}\right\}\right] \\
& +3\left\{2 \log \frac{-p^{2}+\left(\frac{a}{2}\right)^{2} / R^{2}}{-p^{2}}+\log \frac{-p^{2}+a^{2} / R^{2}}{-p^{2}}\right\}-4 N_{\Psi} \log \frac{-p^{2}+\left(\frac{a}{2}\right)^{2} / R^{2}}{-p^{2}} .
\end{aligned}
$$

After summation over $n$ and a Wick rotation with $p^{0}=i p_{E}^{0}$, one gets

$$
\begin{aligned}
V_{\text {eff }}(a)-V_{\text {eff }}(0)= & \frac{1}{2} \int \frac{\mathrm{d}^{4} p_{E}}{(2 \pi)^{4}} 3\left\{2 \log \left(1+\frac{\sin ^{2}\left(\pi \frac{a}{2}\right)}{\sinh ^{2}\left(\pi R p_{E}\right)}\right)+\log \left(1+\frac{\sin ^{2}(\pi a)}{\sinh ^{2}\left(\pi R p_{E}\right)}\right)\right\} \\
& -4 N_{\Psi} \log \left(1+\frac{\sin ^{2}\left(\pi \frac{a}{2}\right)}{\sinh ^{2}\left(\pi R p_{E}\right)}\right),
\end{aligned}
$$

where $p_{E}^{2}$ denotes the Wick-rotated momentum squared, and $p_{E}$ denotes its square root. Note that the above effective potential is finite.

We are interested in the Higgs quartic coupling derived from the effective potential Eq. (A17). We have the formula

$$
\frac{\mathrm{d}^{4}}{\mathrm{~d} a^{4}} \int \frac{\mathrm{d}^{4} p_{E}}{(2 \pi)^{4}} \log \left(1+\frac{\sin ^{2}(\pi a)}{\sinh ^{2}\left(\pi R p_{E}\right)}\right)=\frac{3}{4 \pi^{2}} \frac{1}{R^{4}} \log \left(4 \sin ^{2}(\pi a)\right)
$$

Applying Eq. (A18) to (A17), we find that the quartic coupling for the physical Higgs boson $h$ is derived as

$$
\begin{aligned}
\frac{\mathrm{d}^{4}}{\mathrm{~d} h^{4}} V_{\mathrm{eff}}(a) & =(g R)^{4} \frac{\mathrm{d}^{4}}{\mathrm{~d} a^{4}} V_{\mathrm{eff}}(a) \\
& =\frac{3}{8 \pi^{2}} g^{4}\left\{3 \cdot 2 \cdot \frac{1}{2^{4}} \log \left(4 \sin ^{2}\left(\pi \frac{a}{2}\right)\right)+3 \log \left(4 \sin ^{2}(\pi a)\right)-4 N_{\Psi} \cdot \frac{1}{2^{4}} \log \left(4 \sin ^{2}\left(\pi \frac{a}{2}\right)\right)\right\} .
\end{aligned}
$$

This should be compared with the Higgs quartic coupling derived from the one-loop effective potential of a 4D model that contains $W^{ \pm}$bosons with mass $m=\frac{a}{2} / R, Z$ boson with mass $m=a / R, N_{\Psi}$ Dirac fermions with mass $m=\frac{a}{2} / R$, and a Higgs boson whose quartic coupling vanishes at tree level. In the $4 \mathrm{D}$ model, the Higgs quartic coupling derived from the one-loop effective potential reads

\footnotetext{
${ }^{5}$ In the model of the main text, this fermion gains a vectorlike mass with a $4 \mathrm{D}$ fermion localized at an orbifold fixed point.
} 


$$
\frac{\mathrm{d}^{4}}{\mathrm{~d} h^{4}} V_{\mathrm{pSM} \text { eff }}=\lambda(\mu)+\frac{3}{8 \pi^{2}}\left\{3 \cdot 2\left(\frac{g}{2}\right)^{4} \log \frac{\frac{1}{4} g^{2} h^{2}}{\mu^{2}}+3 g^{4} \log \frac{g^{2} h^{2}}{\mu^{2}}-4 N_{\Psi}\left(\frac{g}{2}\right)^{4} \log \frac{\frac{1}{4} g^{2} h^{2}}{\mu^{2}}\right\},
$$

where $\mu$ denotes the renormalization scale and $\lambda(\mu)$ is the Higgs quartic coupling generated from RG evolutions. In Eq. (A19), when $a \ll 1$ but $\log a$ is still $O(1$ ) (note that $a$ need not correspond to the true Higgs VEV $246 \mathrm{GeV}$ when deriving the matching condition for the Higgs quartic coupling), one can make approximations of $\log \left(4 \sin ^{2}(\pi a)\right) \simeq \log \left(4 \pi^{2} a^{2}\right) \quad$ and $\quad \log \left(4 \sin ^{2}\left(\pi \frac{a}{2}\right)\right) \simeq$ $\log \left(\pi^{2} a^{2}\right)$, while perturbation theory remains valid. Given these approximations, and noting that $h$ in Eq. (A20) is related to $a$ as $h=a /(g R)$, we obtain from the comparison of Eqs. (A19), (A20) the following oneloop matching condition for the Higgs quartic coupling:

$$
\lambda\left(\frac{1}{2 \pi R}\right)=0
$$

In addition to the field content of the above general model, the model of the main text contains extra $U(1)_{V}$ gauge group that is involved in the breaking of $U(1)_{W} \times U(1)_{V} \rightarrow U(1)_{Y}$, and $4 \mathrm{D}$ chiral fermions localized at $y=0$ that mix with 4D-decomposed components of the 5D bulk fermions along the breaking. However, the presence of the $U(1)_{V}$ gauge group and the 4D chiral fermions has negligible impact on the matching condition $\lambda\left(\frac{1}{2 \pi R}\right)=0$, as shown below. The scale of the $U(1)_{W} \times U(1)_{V} \rightarrow U(1)_{Y}$ breaking is given by the VEV of a scalar $\phi$ localized at $y=0$. When coupling constants $A_{i j}, B_{i j}, C_{i j}, E_{i j}$ in Eq. (16) are $O(1)$, we have

$$
M_{\mathrm{vec}} \sim\langle\phi\rangle .
$$

As seen in Sec. III, the model predicts $M_{\text {vec }}<10^{7.5} \mathrm{GeV}$ and $1 /(2 \pi R)>10^{10.2} \mathrm{GeV}$, and hence there is a large hierarchy between $\langle\phi\rangle$ and the compactification scale given by $\langle\phi\rangle /(2 \pi R) \lesssim 10^{-2.7}$. Consequently, the mixing of the $U(1)_{V}$ and $U(1)_{W}$ gauge bosons and that of the $4 \mathrm{D}$ chiral fermions and 4D-decomposed components of the 5D bulk fermions, induced by $\langle\phi\rangle$, change the scale of $\lambda=0$ only by a negligible amount of about $10^{-2.7}$ or below.
[1] D. Buttazzo, G. Degrassi, P. P. Giardino, G. F. Giudice, F. Sala, A. Salvio, and A. Strumia, Investigating the nearcriticality of the Higgs boson, J. High Energy Phys. 12 (2013) 089.

[2] N. Haba, S. Matsumoto, N. Okada, and T. Yamashita, Effective theoretical approach of Gauge-Higgs unification model and its phenomenological applications, J. High Energy Phys. 02 (2006) 073.

[3] N. Haba, S. Matsumoto, N. Okada, and T. Yamashita, Effective potential of Higgs field in warped gauge-Higgs unification, Prog. Theor. Phys. 120, 77 (2008).

[4] J. Carson and N. Okada, $125 \mathrm{GeV}$ Higgs boson mass from 5D gauge-Higgs unification, Prog. Thoer. Exp. Phys. 2018, 033 B03 (2018).

[5] N. Haba, N. Okada, and T. Yamada, Proton decay prediction from a gauge-Higgs unification scenario in five dimensions, Phys. Rev. D 94, 071701(R) (2016).

[6] N.S. Manton, A new six-dimensional approach to the Weinberg-Salam model, Nucl. Phys. B158, 141 (1979).

[7] D. B. Fairlie, Higgs' fields and the determination of the Weinberg angle, Phys. Lett. 82B, 97 (1979).

[8] D. B. Fairlie, Two consistent calculations of the Weinberg angle, J. Phys. G 5, L55 (1979).

[9] Y. Hosotani, Dynamical mass generation by compact extra dimensions, Phys. Lett. 126B, 309 (1983).

[10] Y. Hosotani, Dynamical gauge symmetry breaking as the casimir effect, Phys. Lett. 129B, 193 (1983).
[11] Y. Hosotani, Dynamics of nonintegrable phases and gauge symmetry breaking, Ann. Phys. (N.Y.) 190, 233 (1989).

[12] I. Antoniadis, A possible new dimension at a few TeV, Phys. Lett. B 246, 377 (1990).

[13] I. Antoniadis and K. Benakli, Limits on extra dimensions in orbifold compactifications of superstrings, Phys. Lett. B 326, 69 (1994).

[14] A. Abdalgabar, M. O. Khojali, A. S. Cornell, G. Cacciapaglia, and A. Deandrea, Unification of gauge and Yukawa couplings, Phys. Lett. B 776, 231 (2018).

[15] M. E. Machacek and M. T. Vaughn, Two loop renormalization group equations in a general quantum field theory. 1 . Wave function renormalization, Nucl. Phys. B222, 83 (1983).

[16] M. E. Machacek and M. T. Vaughn, Two loop renormalization group equations in a general quantum field theory. 2 . Yukawa couplings, Nucl. Phys. B236, 221 (1984).

[17] M. E. Machacek and M. T. Vaughn, Two loop renormalization group equations in a general quantum field theory. 3. Scalar quartic couplings, Nucl. Phys. B249, 70 (1985).

[18] A. M. Sirunyan et al. (CMS Collaboration), Measurement of $t \bar{t}$ normalised multi-differential cross sections in pp collisions at $\sqrt{s}=13 \mathrm{TeV}$, and simultaneous determination of the strong coupling strength, top quark pole mass, and parton distribution functions, Eur. Phys. J. C 80, 658 (2020).

[19] G. Aad et al. (ATLAS Collaboration), Measurement of the top-quark mass in $t \bar{t}+1$-jet events collected with the 
ATLAS detector in $p p$ collisions at $\sqrt{s}=8 \mathrm{TeV}$, J. High Energy Phys. 11 (2019) 150.

[20] N. Haba, Y. Mimura, and T. Yamada, Renormalizable $S O(10)$ grand unified theory with suppressed dimension5 proton decays, Prog. Thoer. Exp. Phys. 2021, 023B01 (2021).

[21] B. A. Kniehl, A. F. Pikelner, and O. L. Veretin, mr: A C++ library for the matching and running of the standard model parameters, Comput. Phys. Commun. 206, 84 (2016).

[22] F. Jegerlehner, M. Y. Kalmykov, and O. Veretin, MS versus pole masses of gauge bosons: Electroweak bosonic two loop corrections, Nucl. Phys. B641, 285 (2002).

[23] F. Jegerlehner, M. Y. Kalmykov, and O. Veretin, MS-bar versus pole masses of gauge bosons. 2. Two loop electroweak fermion corrections, Nucl. Phys. B658, 49 (2003).
[24] F. Jegerlehner and M. Y. Kalmykov, O(alpha alpha(s)) correction to the pole mass of the $\mathrm{t}$ quark within the standard model, Nucl. Phys. B676, 365 (2004).

[25] F. Jegerlehner and M. Y. Kalmykov, O(alpha alpha(s)) relation between pole- and MS-bar mass of the $\mathrm{t}$ quark, Acta Phys. Pol. B 34, 5335 (2003).

[26] F. Bezrukov, M. Y. Kalmykov, B. A. Kniehl, and M. Shaposhnikov, Higgs boson mass and new physics, J. High Energy Phys. 10 (2012) 140.

[27] P. Marquard, A. V. Smirnov, V. A. Smirnov, and M. Steinhauser, Quark Mass Relations to Four-Loop Order in Perturbative QCD, Phys. Rev. Lett. 114, 142002 (2015).

[28] B. A. Kniehl, A. F. Pikelner, and O. L. Veretin, Two-loop electroweak threshold corrections in the Standard Model, Nucl. Phys. B896, 19 (2015). 